\title{
Diversification of the restriction-modification system of Streptococcus pyogenes through its acquisition of mobile elements
}

Atsushi Ota ${ }^{1,8}$, Yukiko Nishiuchi ${ }^{2}$, Noriko Nakanishi ${ }^{3}$, Yoshio lijima ${ }^{3}$, Tomotada Iwamoto ${ }^{3}$, Ken Osaki $^{4}$, Yoshitoshi Ogura ${ }^{5}$, Atsushi Toyoda ${ }^{6}$, Yutaka Suzuki ${ }^{7}$, Tetsuya Hayashi ${ }^{5}$, Hiroki Ohge $^{8}$ Hirotada Mori ${ }^{9}$, Manabu Ato ${ }^{10}$, Fumito Maruyama ${ }^{1,11, *}$

${ }^{1}$ Microbial Genomics and Ecology, Office of Academic Research and Industry-Government Collaboration, Academy of Hiroshima University, Higashi-Hiroshima, Hiroshima, 739-8530, Japan

2 Toneyama Institute for Tuberculosis Research Osaka City University Medical School, 5-1-1 Toneyama, Toyonaka, Osaka 560-8552, Japan

${ }^{3}$ Department of Infectious Diseases, Kobe Institute of Health, Kobe, Hyogo 650-0046, Japan 4TOMY DIGITAL BIOLOGY CO., LTD, Taito-Ku, Tokyo 112-0002, Japan

${ }^{5}$ Department of Bacteriology, Faculty of Medical Sciences, Kyushu University, Fukuoka 8128582, Japan

${ }^{6}$ Comparative Genomics Laboratory, National Institute of Genetics, Mishima, Shizuoka 4110801, Japan

${ }^{7}$ Medical Genome Sciences, Graduate School of Frontier Sciences, University of Tokyo, Chiba 277-8562, Japan

8 Department of Infectious Diseases, Hiroshima University Hospital, Kasumi 1-2-3 Minamiku, Hiroshima City, 734-8551, Japan

${ }^{9}$ Data Science Center, Division of Biological Science, Nara Institute of Science and Technology, Nara 630-0192, Japan

10 Department of Immunology, National Institute of Infectious Diseases, Shinjuku-Ku, Tokyo 1628640, Japan

11 Scientific and Technological Bioresource Nucleus, Universidad de La Frontera, Av. Francisco Salazar 01145, Temuco 4811230, Chile

* Corresponding author: Fumito Maruyama

Microbial Genomics and Ecology, Office of Academic Research and Industry-Government Collaboration, Academy of Hiroshima University, Higashi-Hiroshima, Hiroshima, 739-8530, Japan

Tel/Fax: +81-824-24-7048

Email address: fumito@hiroshima-u.ac.jp 


\section{ABSTRACT}

Restriction-modification (RM) systems are typically regarded as "primitive immune systems" in bacteria. The roles of methylation in gene regulation, segregation, and mismatch repair are increasingly recognized. To analyze methyltransferase (MTase) diversity in Streptococcus pyogenes, we compared the RM system distribution in eight new complete genome sequences obtained here and in the database-deposited complete genome sequences of 51 strains. The MTase gene distribution showed that type I MTases often change DNA sequence specificity via switching target recognition domains between strains. The type II MTases in the included strains fell into two groups: a prophage-dominant one and a CRISPR-dominant one. Some highly variable type II MTases were found in the prophage region, suggesting that MTases acquired from phage DNA can generate methylome diversity. Additionally, to investigate the possible contribution of DNA methylation to phenotype, we compared the methylomes and transcriptomes from the four most closely related strains, the results of which suggest that phage-derived methylases possibly regulate the methylome, and, hence, regulate expression levels in $S$. pyogenes. Our findings will benefit further experimental work on the relationship between virulence genes and pathogenicity in S. pyogenes.

\section{INTRODUCTION}

In bacteria, DNA methylation is associated with restriction-modification (RM) systems. RM systems usually encode restriction endonuclease (REase) and DNA methyltransferase (MTase). The REase recognizes and cleaves foreign DNA sequences at specific sites, while MTase activity distinguishes "self" from "non-self" DNA by transferring a methyl from S-adenosyl-methionine to the same specific DNA sequence within the host genome $(1,2)$. The methylated bases found in bacteria are N6-methyl-adenine $(6 \mathrm{~mA})$, C5-methyl-cytosine $(5 \mathrm{mC})$, and N4-methyl-cytosine (4mC) (3-5).

RM systems can be classified into four types (I, II, III, and IV) based on their molecular structure, cofactor requirements, mechanism of action, target sequence, and cleavage position $(2,4,5)$. The most abundant type of RM system is type II (69.5\% of all 26,582 RM systems in 5,568 analyzed bacterial genomes) (6). In addition to having an MTase and REase, some type II RM systems also contain various subclasses of enzymes (e.g., IIB, IIG, IIL, and some IIH) that possess both methylation and endonuclease activity (2). Type I RM systems are the secondmost-abundant RM systems in prokaryotic genomes (17.4\% of all 26,582 RM systems in 5,568 analyzed bacterial genomes) $(6,7)$. Typical multi-subunit-complex type I RM systems contain an REase, MTase, and a specificity domain. These complexes either fully methylate the recognition sites occurring on only one DNA strand or cleave the DNA several bases upstream or downstream of recognition sites that are unmethylated on both strands (2). Type III RM systems are comprised 
of a Mod (MTase) protein, which recognizes asymmetric target sequences, and an REase protein. There are relatively few type III MTases in prokaryotic genomes $(6.8 \%$ of all 26,582 RM systems in 5,568 analyzed bacterial genomes) (7). Finally, type IV RM systems lack methyltransferase activity and consist of only one or two DNA methylation-specific REases.

The roles of the RM system besides its function in the prokaryotic immune system have become more apparent in recent years. The type I specificity subunits of some bacterial species have been found to become switched with one another, thereby producing heterogeneity in gene expression (8-12). This "phase variation" can also contribute to control bacterial virulence, immune evasion, and niche adaptation (13). "Solitary methyltransferases" can also mediate DNA methylation independently of an active cognate REase. Solitary MTase, a type II RM system, has no REase but is nevertheless involved in the global gene regulation of several bacterial species $(14,15)$. Two examples of solitary bacterial MTases are DNA adenosine methyltransferase (Dam) and cell cycle-regulated methyltransferase (CcrM). Dam methylation of GATC sites in Escherichia coli has been shown to regulate the timing of DNA replication, whereas in Caulobacter crescentus, CcrM regulates the cell cycle (16). These studies have extended our understanding, such that we now view DNA methylation as an epigenetic signaling mechanism used by bacteria to help with their ongoing adaptation.

Streptococcus pyogenes causes uncomplicated pharyngitis, scarlet fever, rheumatic fever, and streptococcal toxic shock syndrome (STSS). Invasive strains of $S$. pyogenes are often reported as "flesh-eating" bacteria because infections with these pathogens can progress rapidly and dramatically after illness onset, with an approximate mortality rate of $40 \%$ (18). This is why many researchers study $S$. pyogenes despite it not being a model organism. The incidence of $S$. pyogenes infection has risen since 2011 (17-19), and elucidating its invasion mechanism has become a pressing issue. It is thought that the virulence factors encoded by phages are involved in bacterial virulence to some extent (20). Comparative genome analyses have suggested the pathogenic diversity of $S$. pyogenes might result from single-nucleotide polymorphisms (SNPS) in genes such as those encoding two-component systems (TCSs) (21). However, the presence or absence of some specific genes and SNPs in TCSs were not found to contribute to the pathogenesis widely (22). Thus, other factors, like epigenetic function, should be investigated for their potential to define the pathogenicity of $S$. pyogenes.

To analyze the MTase gene diversity in $S$. pyogenes, the eight $S$. pyogenes strains we sequenced herein were compared with 51 complete genome database-deposited sequences to produce an updated view of the phylogenetic diversity of this species. The results of our methylome and RNA-Seq analyses prompt us to suggest that phage-derived methylases regulate the methylome, and hence, regulate expression levels in S. pyogenes. Our findings contribute to the current knowledge base about the effects of epigenetic modification on the pathological 
diversification of S. pyogenes.

\section{MATERIALS AND METHODS}

\section{Strains and DNA preparation}

For genome sequence determination, we obtained four $S$. pyogenes strains from the American Type Culture Collection (ATCC) (ATCC14918, ATCC14919, MGAS9429, and MGAS2096) and four strains from the Department of Infectious Diseases, Kobe Institute of Health (KIH05-6, KIH0645, KIH06-201, and KIH08-6) (Table 1). ATCC14918 and ATCC14919 were isolated from a human pharynx in 1986. ATCC14919 is an indicator strain lysed by a phage of ATCC14918. MGAS9429 was isolated from an animal swab (23). MGAS2096 was isolated from a patient with acute poststreptococcal glomerulonephritis in 1993 (23). The emm type of all four of these strains is emm12. $\mathrm{KIH05-6,} \mathrm{KIH06-45,} \mathrm{KIH06-201,} \mathrm{and} \mathrm{KIH08-45} \mathrm{were} \mathrm{isolated} \mathrm{in} \mathrm{2005-2008} \mathrm{from} \mathrm{patients} \mathrm{with}$ pharyngitis in Japan, and their serotypes are emm3, emm6, emm6, and emm156, respectively. These strains were cultured in Todd-Hewitt broth (BD Diagnostic Systems; Sparks, MD, USA) supplemented with $0.2 \%$ yeast extract, as previously described (24).

DNA was extracted from these eight $S$. pyogenes isolates, as previously described (24). The extracted DNA concentrations were determined using the Quant-iT PicoGreen dsDNA Assay Kit (Life Technologies Corporation, CA, USA), and DNA quality was checked using a NanoDrop 1000 instrument (Thermo Fisher Scientific, DE, USA). The 51 complete $S$. pyogenes genome sequences from the PATRIC genome online database (http://www.patricbrc.org) were obtained on 11 July 2016 (Supplementary Table S1). They were used as references for the comparative genomics analyses.

\section{SMRT sequencing and sequence annotation}

Complete genome sequences were determined for the strains using the PacBio RSII platform (Pacific Biosciences, Menlo Park, CA, USA), a 20-kb insert-sized library, and P4C2 chemistry (Table 1). De novo assembly was conducted using Falcon 0.5 .0 with the default parameters (25) for strain MGAS9429. Canu ver. 1.2 with the default parameters (26) was used for the other seven strains. Genome annotation was performed by using Prokka ver. 1.11 with the default parameters (27).

\section{Characterizing RM systems}

RM systems were detected using a sequence similarity search (on May 21, 2015) with the RM genes registered in REBASE (http://rebase.neb.com/rebase/rebase.html) (28). Component genes from the RM systems were annotated by BLASTP searching (29) against the protein sequences using the gold-standard library for RM systems obtained from the database as queries. 
All hits were clustered with Blastclust in the NCBI Blast package (29), and the resulting clusters were merged when they showed over $90 \%$ sequence similarity in the alignable regions.

Target recognition domain (TRD) sequences were determined and classified based on the BLASTP results. Sequences lacking similarity in the BLASTP search (e-value: $<1 e^{-5}$ ) against known TRDs in REBASE were classified as new TRD variants. Besides the gold standard, we used information on the methylation patterns of the $S$. pyogenes strains available at REBASE PacBio (http://rebase.neb.com/cgi-bin/pacbio; last accessed March 16, 2016) to predict the recognition sequences of the type I and II RM systems.

\section{Characterizing prophage and CRISPR regions}

Prophages in each genome were predicted using PhiSpy ver. 2.3 (30) run with the default parameters, and the prophage sequences were re-annotated using Prokka ver. 1.11. The prophage region sequences were represented from Easyfig (http://mjsull.github.io/Easyfig/). Each CDS was compared by BLASTN. The percentage average nucleotide identity (ANI) was calculated by applying the ANIm tool in the JSpecies package(31). We also analyzed the number of spacer arrays and prophages in each genome. Acquired CRISPR loci were predicted below.

\section{Determining emm types}

Coding sequences (CDSs) were searched using BLASTN against the emm database in the Centers for Disease Control and Prevention (ftp://ftp.cdc.gov/pub/infectious_diseases/biotech/emmsequ/) (29). CDSs that perfectly matched specific emm genes in the database were considered to be emm genes from the corresponding strain.

\section{Gene content and genome structure analyses}

Pan and core genomes were defined using Roary v3.6 with the default parameters (32). Proteins with similar sequences were clustered using CD-Hit. The BLASTP cut-off value was set at $95 \%$. Core- and pan-genome protein family numbers were estimated by genome sampling up to the number of input genomes at the default setting. The chromosomal locations of these genes were inspected to determine whether they were located in the recombination tracts predicted in a previous report by fastGEAR (33-34).

\section{Constructing phylogenetic trees}

A maximum parsimony phylogenetic tree for the $59 \mathrm{~S}$. pyogenes isolates (51 from databases and eight from this study) was constructed using kSNP3 v3.0 with a k-mer length of 19 (37). The Kchooser script in kSNP3 was used to estimate the optimum k-mer values. Constructed trees 
were visualized using FigTree v1.4.2 (http://tree.bio.ed.ac.uk/software/figtree).

\section{Predicting CRISPR/Cas loci}

CRISPR loci were predicted using the CRISPR Recognition Tool ver. 1.2 with a minimum repeat length of 30 , maximum repeat length of 48 , and maximum spacer length of 80 (38). To characterize the spacer sequences, the spacer list was subjected to a BLASTN search against the following four databases: 1) the "gold standards" dataset of REBASE (accessed October 2016), 2) 59 S. pyogenes genome sequences, 3) predicted RM systems in 59 S. pyogenes strains, and 4) prophage region sequences in $59 \mathrm{~S}$. pyogenes strains. The BLASTP cut-off value was set at $95 \%$ for identity and $90 \%$ for query coverage.

\section{Methylome analysis}

Genomic DNA was sequenced on the PacBio RS II platform. Base modifications were analyzed using the RS_Modification_Detection.1 tool from the SMRT analysis package version 2.3.0. Briefly, after the reads were mapped to the genome sequences, the interpulse durations were measured for all nucleotide positions in the genomes and compared with the expected durations in a kinetic model of the polymerase (39) to identify significant associations. The number of methylated bases in a 1-kb window was counted with a sliding 500-bp window for each strand to overview the methylation distribution.

\section{Transcriptome analysis}

RNA was extracted from three biological replicates per condition. RNA extraction and DNase treatment were performed using the RiboPure-Bacteria kit (Ambion; Austin, TX, USA), and rRNA was depleted with the Illumina Ribo-Zero rRNA Removal kit (Epicentre). RNA integrity was checked with an RNA nano chip (Agilent Technologies, catalog \# 5067-1511) using the Agilent 2100 Bioanalyzer. Sequencing libraries were prepared and barcoded with the SureSelect Strand Specific RNA Library Prep system (Agilent Technologies, catalog \# G9691A and G9691B). The libraries were sequenced with Hiseq3000 to generate 100 base-pair paired-end reads. Read

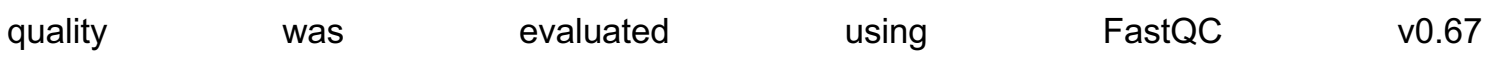
(http://www.bioinformatics.babraham.ac.uk/projects/fastqc/). The sequences were quality filtered and adapter trimmed using Trimmomatic v0.3625 under the default parameters. The reads were mapped to each genome by HISAT run with the default parameters. Mapped reads were visualized by the Integrative Genomics Viewer (IGV) v2.5 (http://www.broadinstitute.org/igv).

\section{RESULTS}

General features of the genome, RM system, and methylome 
We sequenced eight $S$. pyogenes strains with a coverage range of 76-1372. The final assemblies were gathered into each one contig, and determined as complete sequences (Table 1). On average, a total of 1876 genes with 68 tRNA genes and 18 rRNA operons were predicted. We analyzed the whole genome data for all 59 S. pyogenes strains. The pan-genome size and the number of core genes were estimated, and we confirmed the previously proposed assertion (40) that $S$. pyogenes is an open pan-genome species (Supplementary Figure S1). The general characteristics of the genomes are shown in Table 1 and Supplementary Table S1.

We compared the RM systems from all $59 \mathrm{~S}$. pyogenes strains using REBASE, which is a comprehensive and fully curated database of RM systems. We found type I and II RM systems in these 59 S. pyogenes strains (Table 2); none of the strains in the present study had type III or IV systems. Our methylome analysis of the eight newly sequenced strains showed that each strain has different methylation motifs (Table 3). A DNA synthesis kinetics analysis revealed that the predominant modification type was $\mathrm{m} 6 \mathrm{~A}$, whereas other types (e.g., $\mathrm{m} 4 \mathrm{C}$ and $\mathrm{m} 5 \mathrm{C}$ ) were infrequent but did exist (Supplementary Figure S2). By counting the number of methylated bases per 1,000 bases (Supplementary Figure S3), we found that the distribution of methylated bases across the genome differed on each strand. Pathogenesis-related genes, such as the fibronectinbinding protein, were identified in the densely methylated region (Supplementary Table S2). A motif analysis showed that all the motifs targeted by type 1 RMs on each genome were $100 \%$ methylated. In contrast, the other motifs were not. For example, only $51.4 \%$ of 5'-GMGGTAVYR motifs on the ATCC14918 genome were methylated. Finally, we clustered the methylated bases to identify motifs related to recognition sequences (Table 3 ).

\section{Relationship between type I RM systems and genome phylogeny}

The $S$ subunit of a type I RM system contains two tandem TRD sequences, TRD1 and TRD2 (Figure 1). In each TRD pair of the eight strains whose genome sequences were determined here, the combination of TRD1 and TRD2 in the four emm12 strains was the same and recognized GCANNNNNNTTAA (Figure 1, Table 3, and Supplementary Figure S4). Similarly, the TRD combination in the emm6 strains (KIH05-6, KIH08-6) was the same and recognized CAAYNNNNNNTTYG. The emm type differed between KIH06-45 and KIH06-201, but the TRD combination in these strains was the same and recognized GCANNNNNNRTTG.

We compared the maximum parsimony tree constructed using genome-wide SNPs with the type I TRD combination of $S$. pyogenes (Figure 1). With the clades represented by the emm12 strains, the TRD combinations were all $a$ and $b$, meaning that they share the same recognition sequences (Supplementary Figure S4). The TRD combination appears to be mostly consistent with the phylogeny; however, despite being close in phylogeny, the 12_Manfredo strain TRD combination was different from that of the 24_M23ND strain. 


\section{Features and distributions of the type II RM systems}

We identified 127 type II RM systems in the 59 strains, and 104 of the genes from 41 of the strains were solitary MTases (Table 2). The MTase-encoding genes were found in close proximity to the REase gene or both MTase and REase were encoded by a single gene. When no closely located partners were predicted, the MTases were considered solitary MTases. Despite passing quality control and having high genome coverages, the motifs detected in S. pyogenes were not specific (Table 3). Compared with the type I motifs, these candidate type II motifs were not $100 \%$ methylated. For example, in ATCC14918, 51.4\% of the 5'-GMGGTAVYRs were methylated, and $24.6 \%$ of the 5'-RCATAVADs were methylated. Hence, type II RM systems could change the methylome in $S$. pyogenes to a large extent.

We found that some type II MTases are located in the prophage region. As shown in Figure 2, the prophage sequences from four of the emm12 strains were aligned and compared. Strain ATCC14918 contains one prophage, whereas strains ATCC14919, MGAS9429_2, and MGAS2096_2 contain two prophage regions; however, they lack high sequence similarity values despite their close phylogenetic relationship. The prophage regions in ATCC14918 and ATCC14919 share $98 \%$ similarity by ANI (Table 4), but the only prophage in ATCC14918 encodes an MTase. We detected 121 type II MTase genes in the 59 S. pyogenes genomes, with 58 of them encoded in the prophage region as solitary genes (Figure 3). The phylogenetic tree we constructed for the $S$. pyogenes genomes fell into two clades: a prophage-dominant clade and a CRISPR-dominant one. In the prophage-dominant group, the strains were found to contain various MTases, $69 \%$ of which are on prophages. In the CRISPR-dominant group, $31 \%$ of the MTases were found on the prophage. While 19 strains were found to lack a CRISPR-Cas system completely, 18 belong to prophage-dominant groups.

\section{CRISPR spacers exhibiting high nucleotide similarity in the genome}

We identified 317 CRISPR spacers in the 59 isolates. The number of spacers in all the loci varied among the isolates, ranging from 0 to 24 . We obtained unique spacer lists for each CRISPR locus. Nucleotide similarity searches enabled us to also analyze the spacers from the genomes of the $59 \mathrm{~S}$. pyogenes isolates (Supplementary Tables S3-S6). Four predicted MTases (M.Spy19615ORF703P, M.Spy743ORF6920P, M.SpyHSC5ORF7630P, and M.SpyM23NDORF616P) were found to have high similarity with the spacers from ten strains, and two others (M.SpyHSC5ORF7625P and M2.SpySP1ORFDP) have high similarity with the spacers from eight strains. All these type II MTases were encoded on the prophage region rather than in the chromosome. We found that 206 spacers share high nucleotide similarity with six 
MTase sequences from the 59 assessed S. pyogenes strains.

\section{Comparison of the methylation and transcriptome of virulence genes between emm12 strains}

To investigate the possible role of DNA methylation in S. pyogenes, we examined the RNA-Seq results for six virulence genes, speB, spe $C, c s r R, c s r A, m g a$, and $n g a$, in four emm12 strains and conducted a motif analysis. For each region and its upper 20 bases, methylated motifs and mapped reads were compared for both strands (Figure 4). The IGV showed that RNA mapping of $s p e B$ in the four strains clearly differed, with the mRNA expressed on only one strand in ATCC14918. Although the RNA was isolated from the exponential phase, the coverage of each gene differed. The transcriptional level of a region showed no correlation with the location or type of methylated motifs. In ATCC14918 and MGAS9429, csrR and csrA genes are encoded by twocomponent systems.

\section{DISCUSSION}

In bacteria, the number of type I RM systems varies among species, and the RM systems are usually encoded by 5 to 10 genes in the genome (41). For example, in Helicobacter pylori, about 30 type I RM systems are encoded in the chromosomal region of each genome. In contrast, only one or two type I RM system genes are encoded in the S. pyogenes genome, which is a smaller amount than that in other streptococcal species, and these genes are not highly variable (Table 2).

Shuffling of TRDs as "phase variation" has been described in several species over the last 20 years (42-44). One example of phase variation is the switching of TRDs in the type I RM systems of some species, which produces multiple methyltransferase variants and, consequently, variable methylation (11). Another example is type III TRD switching in Haemophilus influenzae, Neisseria gonorrhoeae, and Neisseria meningitidis, where such switching can regulate the expression of multiple genes. Recently, a type I TRD S. pyogenes knockout strain was found to show different methylation and expression profiles from those of the wildtype, but the strain lacked TRD switching, and the phenotype of the knockout was not different from that of the wildtype (45). Hence, the switching of different TRD combinations is considered to make a relatively small contribution to phase variation in S. pyogenes.

In the present study, although the type II MTases of the four emm12 strains were all prophageencoded" solitary MTases", we found that their positions and types were dissimilar. Among our 59 S. pyogenes strains, many of the solitary MTases are on prophages, and the distribution of the type II RM systems is not consistent with the phylogeny (Figure 3). The genome tree was not directly consistent with the distribution of the type II MTases. The clustering determined by 
fastGEAR divided the genomic tree into two groups: a prophage-dominant group and a CRISPRdominant group (Figure 3). In the prophage-dominant group, more MTases tended to be encoded in the prophage region compared with in the CRISPR-dominant group. It is already known that phage can disseminate various genes to bacteria (e.g., virulence genes), and the same may also apply to MTases. The similarity and structure analysis of the prophage region from the four closely-related strains revealed the presence of multiple MTases in this region (Figure 2, Supplementary Figure S5). All these MTases were type II solitary MTases, and type I RM was not encoded. Although the chromosomes are closely related in these four strains, their prophages did not share any sequence similarities (Table 4), suggesting that genetic diversification in $S$. pyogenes has frequently occurred via prophage acquisition.

It is known that the CRISPR spacers in S. pyogenes share sequence similarity with prophage sequences. It is also known that the presence of few CRISPR spacers in the genome increases the chance of $S$. pyogenes acquiring and integrating more prophages (46). This suggests that $S$. pyogenes use CRISPR to control prophage invasion, and this facilitates genetic diversification. In this study, the sequences of some spacers showed high similarity to those of MTases, suggesting that genetic diversification in S. pyogenes has accelerated via acquiring MTase-encoding phages. It is thought that the main reason phages have MTases is to avoid the host defense mechanism, i.e., the RM system, which is incorporated into the host genome. However, it is possible that the host is actively incorporating phages that contain MTase, which in turn can diversify its methylome.

That MTase-targeted spacers exist in bacteria supports the notion that they are involved in an evolutionary arms race between bacteria and phages (47). Similarly, in S. pyogenes, the number of spacers in its genome is negatively correlated with the number of phages (46) (48), and the number of MTases encoded on phages in the prophage-dominant group is also significantly higher than that in the CRISPR-dominant group, suggesting that an evolutionary arms race also exists in S. pyogenes.

Methylome diversification, as driven by changes in the TRD combinations present in a genome, has been reported in some species (41). The present study is the first to analyze the methylase distribution in $S$. pyogenes strains comprehensively. Further analysis of four closely related strains enabled us to identify some virulence factors and various methylation sites (and their sequences) among the strains. Additionally, pathogenesis-related genes, such as the fibronectinbinding protein, were identified in the densely methylated region (Supplementary Table S2), but the biological significance of these instances of hypermethylation is not yet clear. These results suggest that phage-derived methylome diversification may affect the pathogenicity profiles of $S$. pyogenes strains.

An active methylase encoded on a prophage in S. pyogenes has already been reported (49). Expression levels and methylome changes in $S$. pyogenes have also been described quite 
recently (45). The authors of that study elucidated the contribution of the type I RM system to the methylome and phenotype. The present study differs in that the RM systems in multiple $S$. pyogenes strains were comprehensively detected and compared with the methylome. Our methylome analysis was conducted in four closely related strains so that we could compare the transcriptome and methylome analyses. The mRNA expression level of each allele was dissimilar, the type and position of the recognition sequence in the genome also differed (Figure 4), and the four strains fell within the CRISPR-dominant group (Figure 3).

\section{CONCLUSION}

To understand the effect of the RM system as related to genome diversification in S. pyogenes, we acquired and compared the complete genome sequences of $59 \mathrm{~S}$. pyogenes strains. We found that the $S$. pyogenes RM system is diversely distributed in the genome, likely at least partially due to phage-driven gene transfer. Diversification of DNA methylation was also observed in the strains. These findings support the hypothesis that phages can be incorporated into the $S$. pyogenes genome by circumventing the biological defense mechanism of the restriction system by retaining their methylation enzymes. Furthermore, it seems likely that the diversity of DNA modification seen in our strains is regulated by methylases derived from external factors, or phages. It will be necessary to confirm that expression levels and phenotype changes have occurred by using a gene disruption strain in the future. Furthermore, to avoid possible bias relating to the isolation sources of the database strains, the inclusion of additional strains isolated from various situations will be necessary to provide informational and statistical robustness to similar studies. It would also be worthwhile to clarify the effect of DNA modification in S. pyogenes on the disease condition by further analyzing its epigenome under various disease states.

\section{ACCESSION NUMBERS}

The genome sequences described in this study have been deposited in DDBJ/DRA/GenBank under the accession no. DRA004914 (BioProject PRJDB4542). The data described here can be freely and openly accessed in GenBank.

\section{Funding}

This work was supported by Grants-in-Aid for Scientific Research from the Ministry of Education, Culture, Sports, Science and Technology, Japan (grant numbers 221S0002, 16H05501, 18KK0436, and 20H00562) and Japan Agency for Medical Research and Development (AMED) (grant numbers 17fk0108116h0401 and 20fk0108129j0001).

\section{CONFLICT OF INTEREST}


Not applicable.

\section{ACKNOWLEDGMENTS}

We thank Sandra Cheesman, Ph.D., and Katie Oakley, Ph.D., from Edanz Group (www.edanzediting.com/ac) for editing drafts of this manuscript.

\section{REFERENCES}

1. Vasu,K., Vasu,K., Nagaraja,V. and Nagaraja,V. (2013) Diverse functions of restrictionmodification systems in addition to cellular defense. Microbiol. Mol. Biol. Rev., 77, 53-72.

2. Ershova,A.S., Rusinov,I.S., Spirin,S.A., Karyagina,A.S. and Alexeevski,A.V. (2015) Role of restriction-modification systems in prokaryotic evolution and ecology. Biochemistry Mosc., 80, 1373-1386.

3. Donczew,R., Zakrzewska-Czerwińska,J. and Zawilak-Pawlik,A. (2014) Beyond dnaA: the role of DNA topology and DNA methylation in bacterial replication initiation. J. Mol. Biol., 426, 2269-2282.

4. Adhikari,S. and Curtis,P.D. (2016) DNA methyltransferases and epigenetic regulation in bacteria. FEMS Microbiol. Rev., 40, 575-591.

5. Sánchez-Romero,M.A. and Casadesús,J. (2019) The bacterial epigenome. Nat. Rev. Microbiol., 1, 76-14.

6. Oliveira,P.H. and Fang,G. (2020) Conserved DNA methyltransferases: A window into fundamental mechanisms of epigenetic regulation in bacteria. Trends Microbiol., 10.1016/j.tim.2020.04.007.

7. Oliveira,P.H., Touchon,M. and Rocha,E.P.C. (2014) The interplay of restriction-modification systems with mobile genetic elements and their prokaryotic hosts. Nucleic Acids Res., 42, 10618-10631.

8. Manso,A.S., Chai,M.H., Atack,J.M., Furi,L., De Ste Croix,M., Haigh,R., Trappetti,C., Ogunniyi,A.D., Shewell,L.K., Boitano,M., et al. (2014) A random six-phase switch regulates pneumococcal virulence via global epigenetic changes. Nat. Commun., 5, 5055.

9. Furuta,Y., Konno,M., Osaki,T., Yonezawa,H., Ishige,T., Imai,M., Shiwa,Y., Shibata-Hatta,M., Kanesaki,Y., Yoshikawa,H., et al. (2015) Microevolution of virulence-related genes in Helicobacter pylori familial infection. PLoS ONE, 10, e0127197. 
10. Zheng,H., Dietrich,C., Hongoh,Y. and Brune,A. (2016) Restriction-modification systems as mobile genetic elements in the evolution of an intracellular symbiont. Mol. Bio. Evol., 33, $721-725$.

11. Atack,J.M., Tan,A., Bakaletz,L.O., Jennings,M.P. and Seib,K.L. (2018) Phasevarions of bacterial pathogens: Methylomics sheds new light on old enemies. Trends Microbiol., 26, 715-726.

12. Phillips,Z.N., Tram,G., Seib,K.L. and Atack,J.M. (2019) Phase-variable bacterial loci: how bacteria gamble to maximise fitness in changing environments. Biochem. Soc. Trans., 47, $1131-1141$

13. Atack,J.M., Srikhanta,Y.N., Fox,K.L., Jurcisek,J.A., Brockman,K.L., Clark,T.A., Boitano,M., Power,P.M., Jen,F.E.-C., McEwan,A.G., et al. (2015) A biphasic epigenetic switch controls immunoevasion, virulence and niche adaptation in non-typeable Haemophilus influenzae. Nat. Commun., 6, 7828.

14. Fang,G., Munera,D., Friedman,D.I., Mandlik,A., Chao,M.C., Banerjee,O., Feng,Z., Losic,B., Mahajan,M.C., Jabado,O.J., et al. (2012) Genome-wide mapping of methylated adenine residues in pathogenic Escherichia coli using single-molecule real-time sequencing. Nat. Biotechnol., 30, 1232-1239.

15. Murray,I.A., Clark,T.A., Morgan,R.D., Boitano,M., Anton,B.P., Luong,K., Fomenkov,A., Turner,S.W., Korlach,J. and Roberts,R.J. (2012) The methylomes of six bacteria. Nucleic Acids Res., 40, 11450-11462.

16. Low,D.A. and Casadesús,J. (2008) Clocks and switches: bacterial gene regulation by DNA adenine methylation. Curr. Opin. Microbiol., 11, 106-112.

17. Ben Zakour,N.L., Davies,M.R., You,Y., Chen,J.H.K., Forde,B.M., Stanton-Cook,M., Yang,R., Cui,Y., Barnett,T.C., Venturini,C., et al. (2015) Transfer of scarlet fever-associated elements into the group A Streptococcus M1T1 clone. Sci. Rep., 5, 15877.

18. Chalker,V., Jironkin,A., Coelho,J., Al-Shahib,A., Platt,S., Kapatai,G., Daniel,R., Dhami,C., Laranjeira,M., Chambers,T., et al. (2017) Genome analysis following a national increase in scarlet fever in England 2014. BMC Genomics, 18, 224.

19. You,Y., Davies,M.R., Protani,M., Mclntyre,L., Walker,M.J. and Zhang,J. (2018) Scarlet fever epidemic in China caused by Streptococcus pyogenes serotype M12: Epidemiologic and 
molecular analysis. EBioMedicine, 28, 128-135.

20. Hamada,S., Kawabata,S. and Nakagawa,I. (2015) Molecular and genomic characterization of pathogenic traits of group A Streptococcus pyogenes. Proc. Jpn. Acad., Ser. B, Phys. Biol. Sci., 91, 539-559.

21. Kreikemeyer,B., Mclver,K.S. and Podbielski,A. (2003) Virulence factor regulation and regulatory networks in Streptococcus pyogenes and their impact on pathogen-host interactions. Trends Microbiol., 11, 224-232.

22. Kachroo,P., Eraso,J.M., Beres,S.B., Olsen,R.J., Zhu,L., Nasser,W., Bernard,P.E., Cantu,C.C., Saavedra,M.O., Arredondo,M.J., et al. (2019) Integrated analysis of population genomics, transcriptomics and virulence provides novel insights into Streptococcus pyogenes pathogenesis. Nat. Genet., 7, e00403-16.

23. Beres,S.B., Richter,E.W., Nagiec,M.J., Sumby,P., Porcella,S.F., DeLeo,F.R. and Musser,J.M. (2006) Molecular genetic anatomy of inter- and intraserotype variation in the human bacterial pathogen group A Streptococcus. Proc. Natl. Acad. Sci. U.S.A., 103, 7059-7064.

24. Maruyama,F., Kobata,M., Kurokawa,K., Nishida,K., Sakurai,A., Nakano,K., Nomura,R., Kawabata,S., Ooshima,T., Nakai,K., et al. (2009) Comparative genomic analyses of Streptococcus mutans provide insights into chromosomal shuffling and species-specific content. BMC Genomics, 10, 358.

25. Chin,C.-S., Peluso,P., Sedlazeck,F.J., Nattestad,M., Concepcion,G.T., Clum,A., Dunn,C., O'Malley,R., Figueroa-Balderas,R., Morales-Cruz,A., et al. (2016) Phased diploid genome assembly with single-molecule real-time sequencing. Nat. Methods, 13, 1050-1054.

26. Koren,S., Walenz,B.P., Berlin,K., Miller,J.R., Bergman,N.H. and Phillippy,A.M. (2017) Canu: scalable and accurate long-read assembly via adaptive k-mer weighting and repeat separation. Genome Res., 27, 722-736.

27. Seemann,T. (2014) Prokka: rapid prokaryotic genome annotation. Bioinformatics, 30, 20682069.

28. Roberts,R.J., Vincze,T., Posfai,J. and Macelis,D. (2015) REBASE--a database for DNA restriction and modification: enzymes, genes and genomes. Nucleic Acids Res., 43, D2989. 
29. Altschul,S.F., Gish,W., Miller,W., Myers,E.W. and Lipman,D.J. (1990) Basic local alignment search tool. J. Mol. Biol., 215, 403-410.

30. Akhter,S., Aziz,R.K. and Edwards,R.A. (2012) PhiSpy: a novel algorithm for finding prophages in bacterial genomes that combines similarity- and composition-based strategies. Nucleic Acids Res., 40, e126-e126.

31. Richter,M. and Rosselló-Móra,R. (2009) Shifting the genomic gold standard for the prokaryotic species definition. Proc. Natl. Acad. Sci. U.S.A., 106, 19126-19131.

32. Page,A.J., Cummins,C.A., Hunt,M., Wong,V.K., Reuter,S., Holden,M.T.G., Fookes,M., Falush,D., Keane,J.A. and Parkhill,J. (2015) Roary: rapid large-scale prokaryote pan genome analysis. Bioinformatics, 31, 3691-3693.

33. Yano,H., Iwamoto,T., Nishiuchi,Y., Nakajima,C., Starkova,D.A., Mokrousov,I., Narvskaya,O., Yoshida,S., Arikawa,K., Nakanishi,N., et al. (2017) Population structure and local adaptation of MAC lung disease agent Mycobacterium avium subsp. hominissuis. Genome Bio. Evol., 9, 2403-2417.

34. Mostowy,R., Croucher,N.J., Andam,C.P., Corander,J., Hanage,W.P. and Marttinen,P. (2017) Efficient inference of recent and ancestral recombination within bacterial populations. Mol. Bio. Evol., 34, 1167-1182.

35. Gardner,S.N., Slezak,T. and Hall,B.G. (2015) kSNP3.0: SNP detection and phylogenetic analysis of genomes without genome alignment or reference genome. Bioinformatics, 31 , 2877-2878.

36. Bland,C., Ramsey,T.L., Sabree,F., Lowe,M., Brown,K., Kyrpides,N.C. and Hugenholtz,P. (2007) CRISPR recognition tool (CRT): a tool for automatic detection of clustered regularly interspaced palindromic repeats. BMC Bioinformatics, 8, 209.

37. Schadt,E.E., Banerjee,O., Fang,G., Feng,Z., Wong,W.H., Zhang,X., Kislyuk,A., Clark,T.A., Luong,K., Keren-Paz,A., et al. (2013) Modeling kinetic rate variation in third generation DNA sequencing data to detect putative modifications to DNA bases. Genome Res., 23, $129-141$.

38. Maruyama, Fumito, et al. (2016) "Streptococcus pyogenes genomics." Streptococcus pyogenes: Basic biology to clinical manifestations, edited by Joseph J. Ferretti et. al., University of Oklahoma Health Sciences Center. 
39. Furuta,Y., Namba-Fukuyo,H., Shibata,T.F., Nishiyama,T., Shigenobu,S., Suzuki,Y., Sugano,S., Hasebe,M. and Kobayashi,I. (2014) Methylome diversification through changes in DNA methyltransferase sequence specificity. PLoS Genet., 10, e1004272.

40. Furuta,Y. and Kobayashi,I. (2012) Movement of DNA sequence recognition domains between non-orthologous proteins. Nucleic Acids Res., 40, 9218-9232.

41. De Ste Croix,M., Vacca,I., Kwun,M.J., Ralph,J.D., Bentley,S.D., Haigh,R., Croucher,N.J. and Oggioni,M.R. (2017) Phase-variable methylation and epigenetic regulation by type I restriction-modification systems. FEMS Microbiol. Rev., 41, S3-S15.

42. Oliver,M.B., Basu Roy,A., Kumar,R., Lefkowitz,E.J. and Swords,W.E. (2017) Streptococcus pneumoniae TIGR4 phase-locked opacity variants differ in virulence phenotypes. $m S p h e r e$, 2, 1543.

43. Nye,T.M., Jacob,K.M., Holley,E.K., Nevarez,J.M., Dawid,S., Simmons,L.A. and Watson,M.E. (2019) DNA methylation from a type I restriction modification system influences gene expression and virulence in Streptococcus pyogenes. PLoS Pathog., 15, e1007841.

44. Nozawa,T., Furukawa,N., Aikawa,C., Watanabe,T., Haobam,B., Kurokawa,K., Maruyama,F. and Nakagawa,I. (2011) CRISPR inhibition of prophage acquisition in Streptococcus pyogenes. PLoS ONE, 6, e19543.

45. Endo,A., Watanabe,T., Ogata,N., Nozawa,T., Aikawa,C., Arakawa,S., Maruyama,F., Izumi,Y. and Nakagawa,I. (2015) Comparative genome analysis and identification of competitive and cooperative interactions in a polymicrobial disease. ISME J., 9, 629-642.

46. Yamada,S., Shibasaki,M., Murase,K., Watanabe,T., Aikawa,C., Nozawa,T. and Nakagawa,I. (2019) Phylogenetic relationship of prophages is affected by CRISPR selection in Group A Streptococcus. BMC Microbiol., 19, 24.

47. Euler,C.W., Ryan,P.A., Martin,J.M. and Fischetti,V.A. (2007) M.Spyl, a DNA methyltransferase encoded on a mefA chimeric element, modifies the genome of Streptococcus pyogenes. J. Bacteriol., 189, 1044-1054. 
Figure 1. Distribution of type I target recognition domains (TRDs). A genomic tree of $59 \mathrm{~S}$. pyogenes isolates and the TRD combinations for each isolate. The letters $a, b, c, e, f, g$, and $h$ represent the TRD types. The recognition sequences of each type I RM system are shown where they are known. The recognition sequences detected in this study are colored in red.

Figure 2. Sequence comparison of prophage regions integrated on the chromosome in $S$. pyogenes strains ATCC14918, ATCC14919, MGAS9429, and MGAS2096. Genome organization representation from Easyfig (http://mjsull.github.io/Easyfig/). Each CDS was compared by BLASTN. The type II Methylases are shown as red arrow.

Figure 3. Distribution of type II MTases and the numbers of prophages and CRISPR spacers. The vertical column names are the MTases detected in this study. Left: An SNP-based genomic tree and the isolate names are shown on the left. The number of SNPs is shown on each node. The strains that we sequenced in this study are listed in red. Middle: Black boxes indicate the presence of corresponding type II MTases. Red boxes indicate the presence of MTases in the prophage regions. The bar chart shows the number of spacers (blue) and prophages (orange) in each genome. Right: Colors indicate donor lineage (population group) of the recent genomic imports. $S$. pyogenes lineage and recombination were inferred using fastGEAR software (Mostowy et al. 2017).

Figure 4. Integrative Genomics Viewer (IGV) representation of mapped Illumina strand-specific RNA-Seq reads versus the locations of methylated motifs in six loci. The RNA-Seq coverage map shows six gene regions from four emm12 strains. The location of each motif and the mapping coverage are shown on each strand from the four isolates. The coverage range is 0 to 2000 . 
Table 1. The complete genome sequences determined in this study.

\begin{tabular}{|c|c|c|c|c|c|c|c|c|c|c|c|c|c|}
\hline Strain & emm type & Isolation source & Accession \# & $\begin{array}{l}\# \text { of } \\
\text { contig }\end{array}$ & Total read length & Genome size & $\begin{array}{l}\text { Genome } \\
\text { coverage }\end{array}$ & $\mathrm{n} 50$ & $\begin{array}{l}\text { GC content } \\
(\%)\end{array}$ & gene & CDS & tRNA & rRNA \\
\hline ATCC14918 & 229 & Pharynx & DRX060373 & 1 & $2,259,100,157$ & $1,796,820$ & $1,257.3$ & $1,796,820$ & 38.56 & 1,768 & 1,682 & 67 & 18 \\
\hline ATCC14919 & 229 & Pharynx & DRX060374 & 1 & $2,411,644,106$ & $1,757,745$ & $1,372.0$ & $1,757,745$ & 38.56 & 1,709 & 1,623 & 67 & 18 \\
\hline MGAS9429_2 & 229 & Pharyngeal swab - animal & DRX060376 & 1 & $2,010,397,268$ & $1,836,296$ & $1,094.8$ & $1,836,296$ & 38.55 & 1,824 & 1,738 & 67 & 18 \\
\hline MGAS2096_2 & 229 & Human clinical specimen & DRX060375 & 1 & $1,628,396,143$ & $1,860,246$ & 875.4 & $1,860,246$ & 38.73 & 1,824 & 1,738 & 67 & 18 \\
\hline KIH05-6 & 6 & pharyngitis & DRX173148 & 1 & $311,592,050$ & $1,866,843$ & 166.9 & $1,866,843$ & 38.58 & 1,914 & 1,828 & 67 & 18 \\
\hline KIH06-45 & 3 & pharyngitis & DRX173149 & 1 & $315,098,671$ & $1,906,193$ & 165.3 & $1,906,193$ & 38.62 & 1,979 & 1,880 & 77 & 21 \\
\hline KIH06-201 & 156 & pharyngitis & DRX173150 & 1 & $152,246,458$ & $1,982,968$ & 76.8 & $1,982,968$ & 38.5 & 2,023 & 1,937 & 67 & 18 \\
\hline KIH08-6 & 6 & pharyngitis & DRX173151 & 1 & $610,902,547$ & $1,905,820$ & 320.5 & $1,905,820$ & 38.6 & 1,969 & 1,883 & 67 & 18 \\
\hline
\end{tabular}


bioRxiv preprint doi: https://doi.org/10.1101/2020.06.30.179317; this version posted July 3, 2020. The copyright holder for this preprint (which was not certified by peer review) is the author/funder, who has granted bioRxiv a license to display the preprint in perpetuity. It is made available under aCC-BY 4.0 International license.

Table 2. Distribution of the RM systems in the $S$. pyogenes strains used in this study.

\begin{tabular}{|c|c|c|c|c|c|c|c|c|}
\hline \multirow[t]{2}{*}{ Type } & \multicolumn{3}{|c|}{ I } & \multicolumn{2}{|c|}{ II } & \multicolumn{2}{|c|}{ III } & \multirow{2}{*}{$\begin{array}{c}\text { IV } \\
\mathrm{R} \\
\end{array}$} \\
\hline & $\mathrm{R}$ & $\mathrm{M}$ & $\mathrm{S}$ & $\mathrm{R}$ & M & $\mathrm{R}$ & M & \\
\hline 01_SF370 & 1 & 3 & 2 & 0 & 2 & 0 & 0 & 0 \\
\hline 02_MGAS8232 & 1 & 2 & 2 & 1 & 5 & 0 & 0 & 0 \\
\hline 03_MGAS315 & 1 & 2 & 2 & 1 & 2 & 0 & 0 & 0 \\
\hline 04_SSI1 & 1 & 2 & 2 & 1 & 2 & 0 & 0 & 0 \\
\hline 05_MGAS10394 & 1 & 1 & 2 & 2 & 3 & 0 & 0 & 0 \\
\hline 06_MGAS6180 & 1 & 2 & 2 & 1 & 2 & 0 & 0 & 0 \\
\hline 07_MGAS5005 & 1 & 3 & 2 & 0 & 2 & 0 & 0 & 0 \\
\hline 08_MGAS9429 & 1 & 1 & 2 & 0 & 3 & 0 & 0 & 0 \\
\hline 09_MGAS 10270 & 1 & 1 & 2 & 1 & 5 & 0 & 0 & 0 \\
\hline 10_MGAS2096 & 1 & 2 & 2 & 0 & 5 & 0 & 0 & 0 \\
\hline 11_MGAS10750 & 1 & 2 & 2 & 0 & 4 & 0 & 0 & 0 \\
\hline 12_Manfredo & 1 & 3 & 2 & 0 & 5 & 0 & 0 & 0 \\
\hline 13_NZ131 & 1 & 1 & 2 & 0 & 1 & 0 & 0 & 0 \\
\hline 14_Alab49 & 1 & 2 & 2 & 0 & 1 & 0 & 0 & 0 \\
\hline 15_MGAS15252 & 1 & 1 & 2 & 0 & 1 & 0 & 0 & 0 \\
\hline 16_MGAS1882 & 1 & 2 & 2 & 1 & 1 & 0 & 0 & 0 \\
\hline 17_M1_476 & 1 & 3 & 2 & 0 & 2 & 0 & 0 & 0 \\
\hline 18_A20 & 1 & 3 & 2 & 0 & 2 & 0 & 0 & 0 \\
\hline 19_HSC5 & 1 & 3 & 2 & 0 & 3 & 0 & 0 & 0 \\
\hline 20_H293 & 1 & 1 & 2 & 0 & 1 & 0 & 0 & 0 \\
\hline 21_STAB902 & 1 & 2 & 2 & 1 & 2 & 0 & 0 & 0 \\
\hline 22_STAB901 & 1 & 2 & 3 & 0 & 2 & 0 & 0 & 0 \\
\hline 23_ATCC19615 & 1 & 2 & 2 & 1 & 1 & 0 & 0 & 0 \\
\hline 24_M23ND & 1 & 1 & 2 & 2 & 3 & 0 & 0 & 0 \\
\hline $25 \_7 \mathrm{~F} 7$ & 1 & 1 & 2 & 0 & 0 & 0 & 0 & 0 \\
\hline 26_HKU360 & 1 & 2 & 2 & 0 & 5 & 0 & 0 & 0 \\
\hline $27 \_10$ & 1 & 2 & 2 & 0 & 2 & 0 & 0 & 0 \\
\hline 28_MTB313 & 1 & 1 & 1 & 0 & 1 & 0 & 0 & 0 \\
\hline 29_MTB314 & 1 & 1 & 1 & 0 & 1 & 0 & 0 & 0 \\
\hline 30_JRS4 & 1 & 2 & 2 & 0 & 1 & 0 & 0 & 0 \\
\hline 31_AP1 & 1 & 4 & 2 & 0 & 3 & 0 & 0 & 0 \\
\hline 32_JRS4 & 1 & 2 & 2 & 0 & 1 & 0 & 0 & 0 \\
\hline 33_D471 & 1 & 2 & 2 & 0 & 1 & 0 & 0 & 0 \\
\hline 34_NGAS743 & 1 & 4 & 2 & 2 & 5 & 0 & 0 & 0 \\
\hline 35_NGAS596 & 1 & 1 & 2 & 0 & 1 & 0 & 0 & 0 \\
\hline 36_NGAS327 & 1 & 1 & 2 & 0 & 0 & 0 & 0 & 0 \\
\hline 37_M28PF1 & 1 & 2 & 2 & 1 & 2 & 0 & 0 & 0 \\
\hline $38 \_5448$ & 1 & 3 & 2 & 0 & 2 & 0 & 0 & 0 \\
\hline 39_STAB10015 & 1 & 2 & 2 & 1 & 2 & 0 & 0 & 0 \\
\hline 40_HKU488 & 1 & 4 & 2 & 0 & 2 & 0 & 0 & 0 \\
\hline 41_NGAS322 & 1 & 1 & 2 & 1 & 4 & 0 & 0 & 0 \\
\hline 42_NGAS638 & 1 & 1 & 2 & 0 & 1 & 0 & 0 & 0 \\
\hline 43_MEW427 & 1 & 1 & 2 & 0 & 0 & 0 & 0 & 0 \\
\hline 44_MEW123 & 1 & 2 & 2 & 0 & 1 & 0 & 0 & 0 \\
\hline 45_FDAARGOS_149 & 1 & 2 & 2 & 0 & 2 & 0 & 0 & 0 \\
\hline 46_AP53 & 1 & 2 & 2 & 0 & 2 & 0 & 0 & 0 \\
\hline 47_MGAS11027 & 1 & 2 & 2 & 0 & 4 & 0 & 0 & 0 \\
\hline 48_MGAS23530 & 1 & 1 & 2 & 0 & 1 & 0 & 0 & 0 \\
\hline 49_MGAS27061 & 1 & 2 & 2 & 0 & 1 & 0 & 0 & 0 \\
\hline 50_NS53 & 1 & 1 & 1 & 0 & 1 & 0 & 0 & 0 \\
\hline 51_STAB09014 & 1 & 2 & 2 & 0 & 1 & 0 & 0 & 0 \\
\hline 52_ATCC14918 & 1 & 1 & 2 & 0 & 1 & 0 & 0 & 0 \\
\hline 53_ATCC14919 & 1 & 1 & 2 & 0 & 2 & 0 & 0 & 0 \\
\hline 54_MGAS9429_2 & 1 & 1 & 2 & 0 & 1 & 0 & 0 & 0 \\
\hline 55_MGAS2096_2 & 1 & 2 & 2 & 0 & 5 & 0 & 0 & 0 \\
\hline 56_KIH05_6 & 1 & 2 & 2 & 1 & 3 & 0 & 0 & 0 \\
\hline 57_KIH06_45 & 1 & 2 & 2 & 1 & 2 & 0 & 0 & 0 \\
\hline 58_KIH06_201 & 1 & 2 & 2 & 2 & 3 & 0 & 0 & 0 \\
\hline 59_KIH08_6 & 1 & 2 & 2 & 2 & 3 & 0 & 0 & 0 \\
\hline
\end{tabular}

R., restriction subunit; M, modification subunit; S, specificity subunit. 
Table 3. The methylation motifs in each strain.

\begin{tabular}{|c|c|c|c|c|c|c|}
\hline Strain & Motif & $\begin{array}{c}\text { Modification } \\
\text { Type } \\
\end{array}$ & $\begin{array}{c}\% \text { of motifs } \\
\text { detected }\end{array}$ & $\begin{array}{c}\text { No. of motifs } \\
\text { detected }\end{array}$ & $\begin{array}{l}\text { No. of motifs } \\
\text { in genome }\end{array}$ & Comments \\
\hline \multirow[t]{4}{*}{ ATCC14918 } & 5'-GCÁNNNNNNTTAA & $\mathrm{m} 6 \mathrm{~A}$ & $100.0 \%$ & 339 & 339 & Complement with TTAANNNNNNTGC \\
\hline & 5'-TT $\underline{A} A N N N N N N T G C$ & $\mathrm{~m} 6 \mathrm{~A}$ & $100.0 \%$ & 339 & 339 & \\
\hline & 5'-GMGGTAVYR & modified_base & $51.4 \%$ & 89 & 173 & \\
\hline & 5'-RCATAVAD & modified base & $24.6 \%$ & 213 & 867 & \\
\hline \multirow[t]{3}{*}{ ATCC14919 } & 5'-GCANNNNNNNTTAA & $\mathrm{m} 6 \mathrm{~A}$ & $100.0 \%$ & 332 & 332 & Complement with TTAANNNNNNTGC \\
\hline & 5'-TT $\underline{A} A N N N N N N T G C$ & m6A & $100.0 \%$ & 332 & 332 & \\
\hline & 5'-AGGAAATTT & m6A & $48.4 \%$ & 44 & 91 & \\
\hline \multirow[t]{4}{*}{ MGAS9429 } & 5'-GCANNNNNNNTTAA & $\mathrm{m} 6 \mathrm{~A}$ & $100.0 \%$ & 342 & 342 & Complement with TTAANNNNNNTGC \\
\hline & 5'-TTÁANNNNNNTGC & $\mathrm{m} 6 \mathrm{~A}$ & $100.0 \%$ & 342 & 342 & \\
\hline & 5'-CGCGGTMNYA & modified_base & $94.1 \%$ & 16 & 17 & \\
\hline & 5'-GMGGTAMYD & modified base & $44.4 \%$ & 95 & 214 & \\
\hline \multirow[t]{5}{*}{ NGAS2096 } & 5'-GCÁNNNNNNTTAA & $\mathrm{m} 6 \mathrm{~A}$ & $100.0 \%$ & 350 & 350 & Complement with TTAANNNNNNTGC \\
\hline & 5'-TT吕ANNNNNNTGC & m6A & $100.0 \%$ & 350 & 350 & \\
\hline & 5'-CNGCATANGNTNNNA & modified_base & $92.9 \%$ & 13 & 14 & \\
\hline & 5'-RCATAVAD & modified_base & $29.4 \%$ & 267 & 908 & \\
\hline & 5'-DNGGTANH & modified base & $6.7 \%$ & 573 & 8561 & \\
\hline \multirow[t]{2}{*}{ KIH05-6 } & 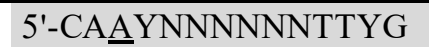 & $\mathrm{m} 6 \mathrm{~A}$ & $99.6 \%$ & 463 & 465 & CRAANNNNNNRTTG \\
\hline & 5'-CRAANNNNNNRTTG & $\mathrm{m} 6 \mathrm{~A}$ & $99.1 \%$ & 461 & 465 & CAAYNNNNNNTTYG \\
\hline \multirow[t]{2}{*}{ KIH06-45 } & 5'-GCANNNNNNRTTG & $\mathrm{m} 6 \mathrm{~A}$ & $98.7 \%$ & 514 & 521 & CAAYNNNNNNTGC \\
\hline & 5'-CAAYYNNNNNNTGC & $\mathrm{m} 6 \mathrm{~A}$ & $98.7 \%$ & 514 & 521 & GCANNNNNNRTTG \\
\hline \multirow[t]{2}{*}{ KIH06-201 } & 5'-GCÁANNNNNRTTG & $\mathrm{m} 6 \mathrm{~A}$ & $90.2 \%$ & 506 & 561 & CAAYNNNNNNTGC \\
\hline & 5'-CAAYNNNNNNTGC & $\mathrm{m} 6 \mathrm{~A}$ & $86.8 \%$ & 487 & 561 & GCANNNNNNRTTG \\
\hline \multirow[t]{2}{*}{ KIH08-6 } & 5'-CRAAANNNNNNRTTG & $\mathrm{m} 6 \mathrm{~A}$ & $98.9 \%$ & 462 & 467 & CAAYNNNNNNTTYG \\
\hline & 5'-CAAYNNNNNNTTYG & $\mathrm{m} 6 \mathrm{~A}$ & $98.5 \%$ & 460 & 467 & CRAANNNNNNRTTG \\
\hline
\end{tabular}

*Methylated bases are underlined. 
Table 4. Average Nucleotide Identity (ANI) (\%) based on prophage sequences.

\begin{tabular}{|c|c|c|c|c|c|c|}
\hline ANIb & ATCC14918-Ф1 & ATCC14919- $\Phi 1$ & MGAS9429 2- $\Phi 1$ & MGAS $9429 \quad 2-\Phi 2$ & MGAS2096 2- $\Phi 1$ & MGAS2096 2- $\Phi 2$ \\
\hline ATCC14918- 11 & --- & 96.56 & 100 & $\mathrm{NaN}$ & 91.06 & 96.56 \\
\hline ATCC 14919- $\Phi 1$ & 96.38 & --_- & 96.38 & $\mathrm{NaN}$ & 99.46 & 100 \\
\hline MGAS9429 2- $\Phi 1$ & 100 & 96.56 & --- & $\mathrm{NaN}$ & 91.06 & 96.56 \\
\hline 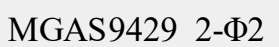 & $\mathrm{NaN}$ & $\mathrm{NaN}$ & $\mathrm{NaN}$ & --- & $\mathrm{NaN}$ & 77.59 \\
\hline MGAS2096 2-Ф1 & 88.32 & 100 & 88.32 & 80.41 & .-- & 100 \\
\hline MGAS2096 2-Ф2 & 96.29 & 100 & 96.29 & 77.01 & 100 & --- \\
\hline
\end{tabular}




\section{Figure 1.}

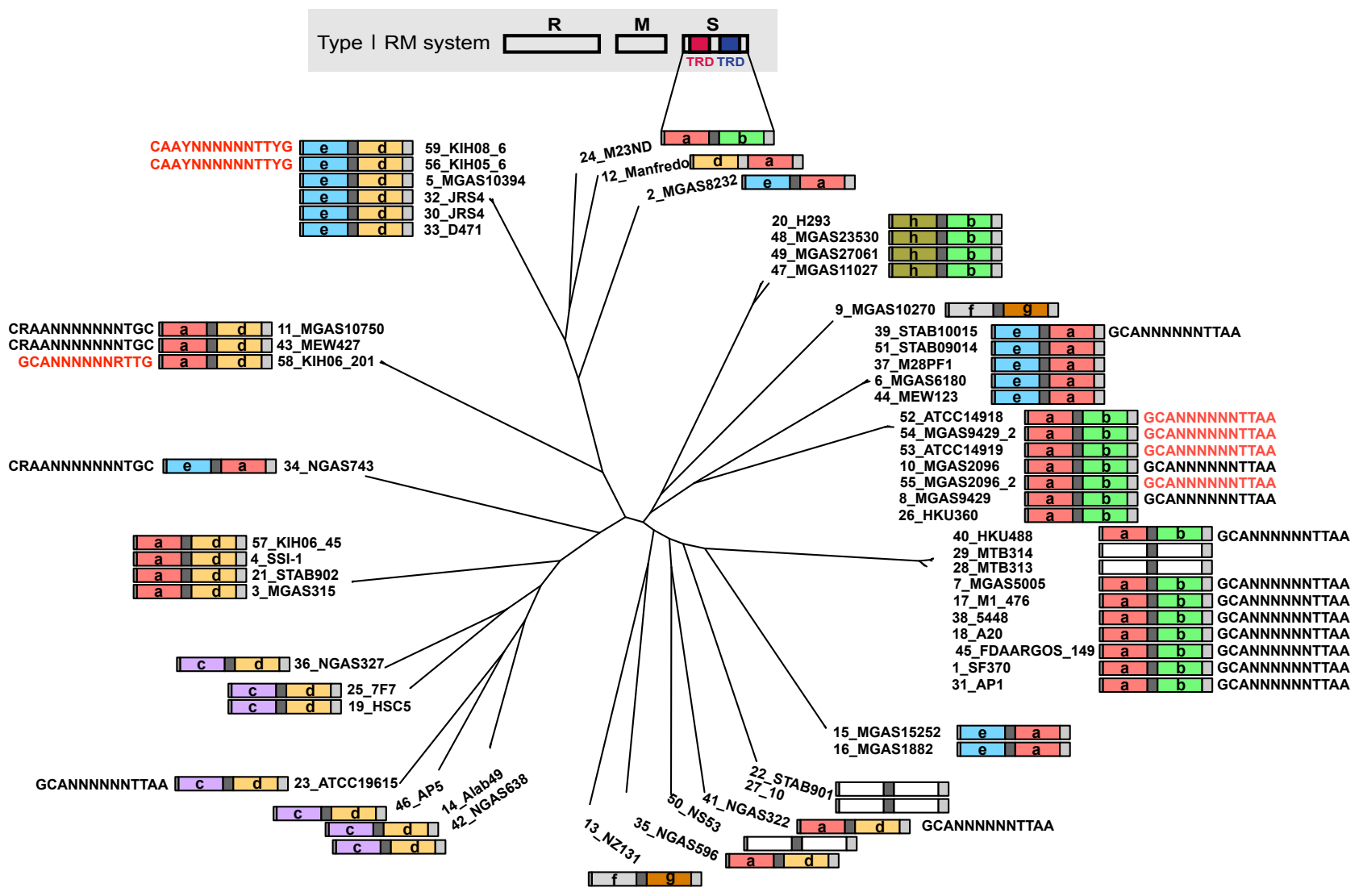




\section{Figure 2.}

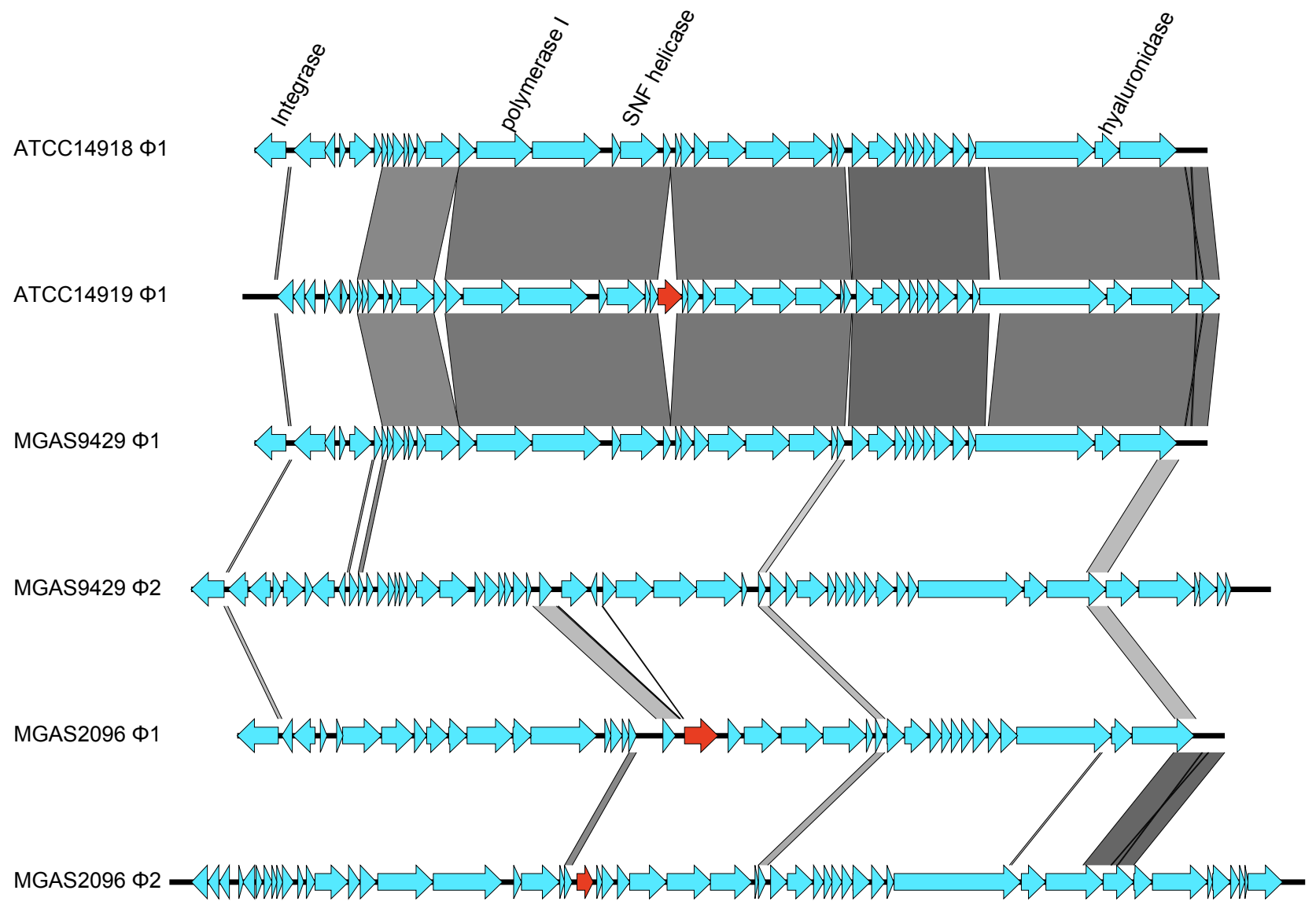


Figure 3.

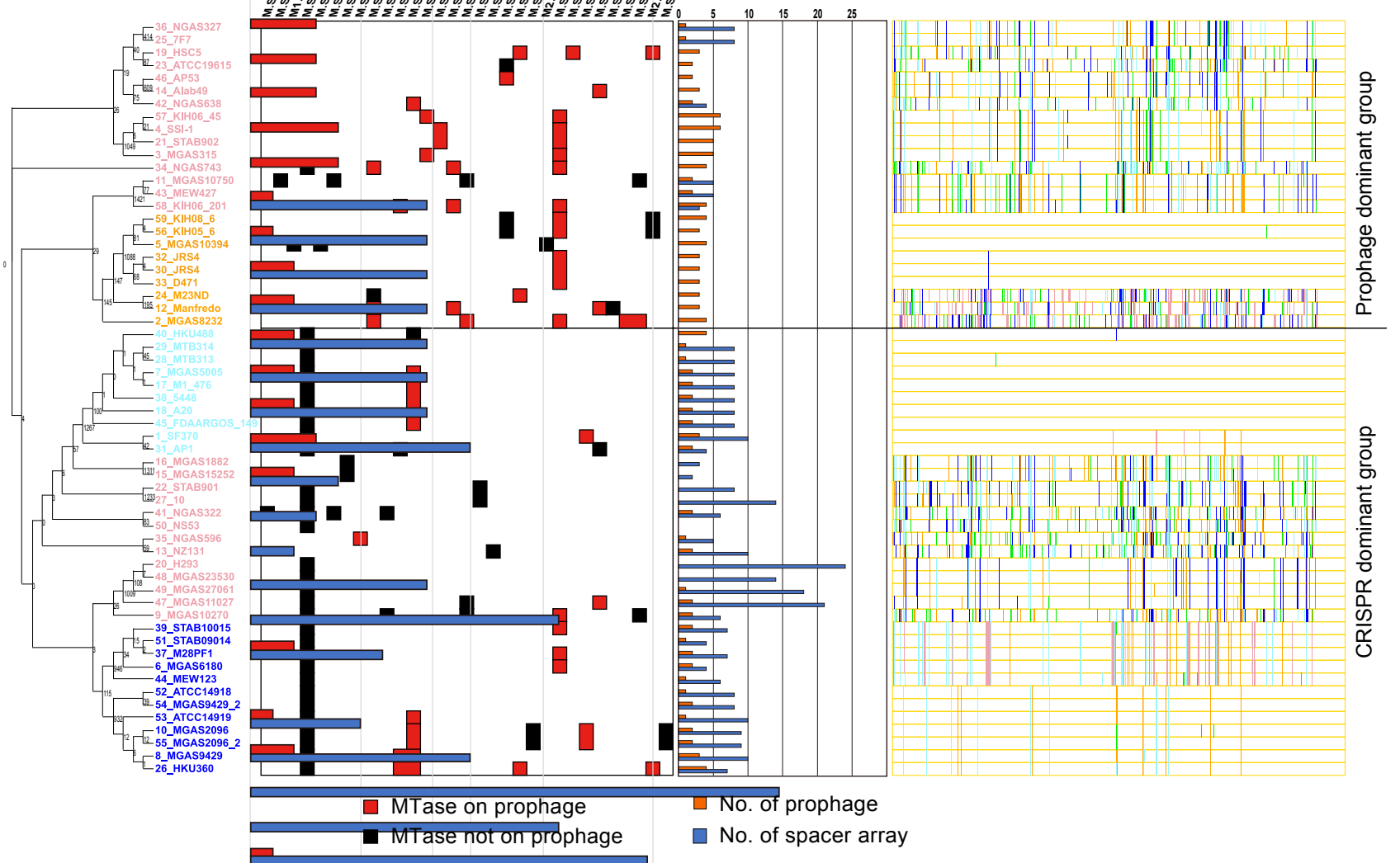




\section{Figure 4.}

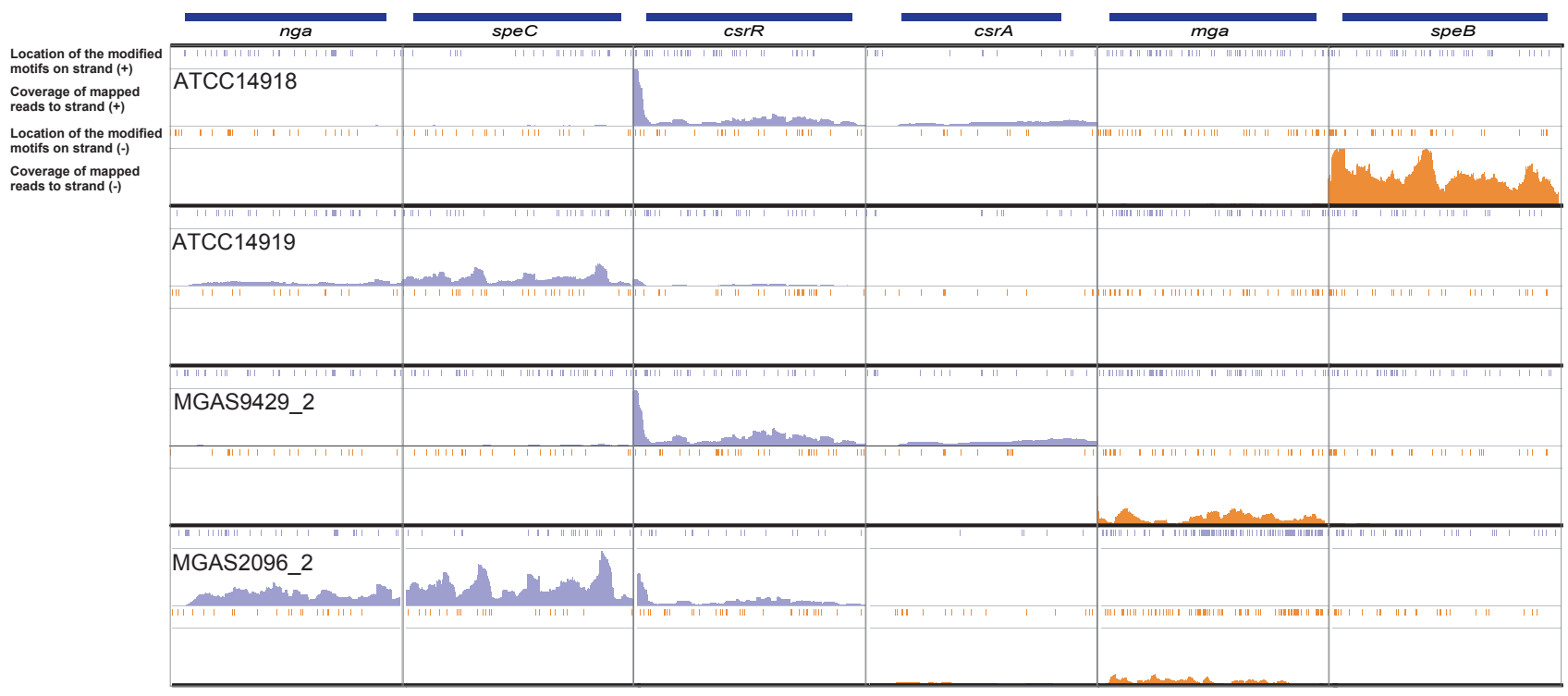

